\title{
New Synthesis of Parallel-Coupled Line Bandpass Filters With Chebyshev Responses
}

\author{
Kuo-Sheng Chin, Member, IEEE, Yi-Chyun Chiou, Student Member, IEEE, and Jen-Tsai Kuo, Senior Member, IEEE
}

\begin{abstract}
Parallel-coupled line filters with Chebyshev passbands are synthesized based on the insertion-loss functions derived by converting the composite $A B C D$ matrices of all coupled stages. With the synthesis, both the electromagnetic simulation and measurement results have accurate in-band response and bandwidth. Simultaneous equations for Chebyshev filters of order $N \leq 9$ are derived for determining geometric parameters of the coupled stages. The tradeoff between circuit bandwidth and ripple level is discussed. In experiments, three planar or quasi-planar structures, including the coupled-line with aperture in the ground plane, the three-line microstrip, and the broadside-coupled lines, are employed to realize the end stages for implementing the high coupling levels. Experimental filters of order up to nine with fractional bandwidth of $30 \%$ or $40 \%$ are fabricated and measured. The results show good agreement with predictions by the theory and simulation.
\end{abstract}

Index Terms-Bandpass filters, Chebyshev response, insertionloss function, parallel-coupled line filter, strong coupling.

\section{INTRODUCTION}

$\mathbf{T}$ HE parallel-coupled configuration has been widely used for realization of microstrip bandpass filters [1]-[4]. Approximate formulas have been well documented for determining dimensions of the coupled stages [3], [4]. These approximate formulas can provide accurate results when the filter order is low and the designated bandwidth is small. When the circuit order is higher or the bandwidth is increased, however, the bandwidth of the synthesized filter will be less than specification. For example, fifth-order filters have bandwidths of only $25 \%$ and $31 \%$ when designated bandwidths are $30 \%$ and $40 \%$, respectively. The bandwidth decrement deteriorates as the designated bandwidth or filter order is increased [5]. The bandwidth decrement is due to the use of the equivalence of a coupled stage to two quarter-wave lines with a frequency-independent $J$-inverter in between [3], [4]. It is noted that equivalence of a distributed network to a lumped element must have a limited bandwidth.

Various methods have been proposed for synthesizing bandpass filters with accurate passband responses. For example, the

Manuscript received November 7, 2007; revised April 17, 2008. First published June 13, 2008; last published July 9, 2008 (projected). This work was supported in part by the Ministry of Education (MoE) under the ATU Plan and National Science Council, Taiwan, R.O.C., under Grant NSC 96-2221-E-182002, Grant NSC 96-2752-E-009-003-PAE, and Grant NSC 95-2221-E-009-037MY2.

K.-S. Chin is with the Department of Electronic Engineering, Chang Gung University, Taoyuan 333, Taiwan, R.O.C. (e-mail: kschin@ mail.cgu.edu.tw).

Y.-C. Chiou and J.-T. Kuo are with the Department of Communication Engineering, National Chiao Tung University, Hsinchu 300, Taiwan, R.O.C. (e-mail: jtkuo@mail.nctu.edu.tw).

Color versions of one or more of the figures in this paper are available online at http://ieeexplore.ieee.org.

Digital Object Identifier 10.1109/TMTT.2008.925572
$Q$ distribution method [6] provides solutions to quarter-wavelength-coupled structures and the insertion-loss function method activates the syntheses of short-circuited quarter-wave stub filters [7] and the parallel-coupled line filters [8]. Note that the circuits in [6]-[8] are limited to maximally flat responses.

Chebyshev filters are more flexible than the maximally flat type due to the degree of freedom for the tradeoff between the passband ripple level and rejection rate in the transition band. This paper extends our previous study on the maximally flat type in [8] to the synthesis of Chebyshev filters of higher orders. Simultaneous equations for solving the geometric parameters of all coupled stages are derived based on the composite $A B C D$ matrices, which take the full distributed property of each stage into account. It will be shown that the coupled stages, especially the end stages, require higher coupling levels than those of the conventional synthesis [3], [4]. In [8], tapped-line input/output is used to realize the high coupling since the gap size or linewidth of the end stages exceeds the resolution limit of the standard printed circuit board (PCB) technology. In this paper, the tapped-line structure is purposely bypassed and the end sections are realized by three substitutes for exploring their performances for implementing the high coupling levels. The first one is the coupled section with an aperture in the ground plane [9]. Enhanced coupling can be obtained by removing the conductor in the ground plane under the section. The second is the three-line microstrip [10]. When it is used to replace a usual coupled-line stage, for a given coupling level, some case studies show that the tight line spacing can be relaxed by six to seven times. The last one is the microstrip-to-coplanar waveguide (CPW) broadside-coupled structure. This structure is well suitable for providing high coupling over a wide bandwidth. The ultra-wide bandwidth bandpass filter in [11] is a typical example. Based on this structure, some miniaturized ring devices [12], [13] have been developed.

This paper puts emphasis on the extension from [8] as follows. First, for Chebyshev passbands, simultaneous equations for determining the modal characteristic impedances of each coupled stage are derived for circuits of order up to nine, in contrast to the sixth-order Butterworth filters in [8]. The ripple level condition is then formulated and the tradeoff between the ripple level and bandwidth is discussed. Finally, the three structures with enhanced coupling are incorporated into the experiments, and measured responses are presented for validating the theory and electromagnetic (EM) simulation.

\section{Chebyshev PAssbands}

An $N$ th-order parallel-coupled line filter consists of a cascade of $N+1$ coupled stages is shown in Fig. 1 . Herein, both the source and load impedances are assumed identical to the 


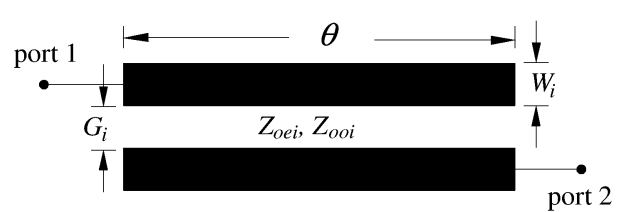

Fig. 1. $i$ th coupled stage with linewidth $W_{i}$ and gap $G_{i}$.

system impedance $Z_{o}$. The $A B C D$ matrix of the $i$ th stage can be expressed as [4], [8]

$$
\left[\begin{array}{cc}
A_{i} & B_{i} \\
C_{i} & D_{i}
\end{array}\right]=\frac{\sin \theta}{T_{i}}\left[\begin{array}{cc}
q S_{i} & \frac{j}{2}\left[T_{i}^{2}+q^{2}\left(T_{i}^{2}-S_{i}^{2}\right)\right] \\
2 j & q S_{i}
\end{array}\right]
$$

where $j=\sqrt{-1}, \theta$ is its electrical length, and $q=\cot \theta, S_{i}=$ $\left(Z_{o e i}+Z_{o o i}\right) / Z_{o}, T_{i}=\left(Z_{o e i}-Z_{o o i}\right) / Z_{o}$, and $Z_{o e i}$ and $Z_{o o i}$ are, respectively, the even- and odd-mode characteristic impedances of the coupled lines. The modal phase velocities of all coupled-lines are assumed identical.

It has been shown in [8] that the entries of the total $A B C D$ matrix of the filter are polynomials of $q$ and the coefficients are functions of $S_{i}$ and $T_{i}$. The insertion loss function can be written as [3]

$$
\frac{P_{o}}{P_{L}}=1-\frac{(B-C)^{2}}{4}
$$

where $P_{o}$ is power available from the source and $P_{L}$ is power delivered to the load. On the other hand, the insertion-loss function of an $N$ th-order Chebyshev filter is defined as [3], [4]

$$
\frac{P_{o}}{P_{L}}=1+\epsilon^{2}\left[\mathbf{T}_{N}(x)\right]^{2}
$$

where $1+\varepsilon^{2} \equiv R$ defines the ripple level and $\mathbf{T}_{N}(x)$ is the Chebyshev polynomial of the first kind of order $N$. The variables $Z_{o e i}$ and $Z_{o o i}$ and, hence, the geometry parameters $W_{i}$ and $G_{i}$, will be solved by matching (2) with (3).

\section{A. Second-Order Filters}

In this section, synthesis of a second-order Chebyshev filter is demonstrated. Due to structural symmetry, we have $S_{1}=S_{3}$ and $T_{1}=T_{3}$. It can be derived that

$$
\begin{aligned}
\frac{j(B-C)}{2}=\frac{1}{2 T_{1}^{2} T_{2} \sin \theta} \times[ & -h_{1}+\cos ^{2} \theta\left(2 h_{1}-h_{2}\right) \\
& \left.+\cos ^{4} \theta\left(-h_{1}+h_{2}-h_{3}\right)\right]
\end{aligned}
$$

where

$$
\begin{aligned}
& h_{1}=2 T_{2}^{2}-\frac{T_{1}^{4}}{2} \\
& h_{2}=S_{1} T_{1}^{2}\left(S_{1}+S_{2}\right)-2\left(S_{1}+S_{2}\right)^{2}-T_{1}^{4}+\frac{S_{1}^{2} T_{2}^{2}}{2}+2 T_{2}^{2} \\
& h_{3}=S_{1} T_{1}^{2}\left(S_{1}+S_{2}\right)-\frac{S_{1}^{2}}{2}\left(S_{1}+S_{2}\right)^{2}-\frac{T_{1}^{4}}{2}+\frac{S_{1}^{2} T_{2}^{2}}{2} .
\end{aligned}
$$

Substituting (4a) into (2), we have

$$
\begin{aligned}
\frac{P_{o}}{P_{L}}=1 & +\left[\frac{1}{2 T_{1}^{2} T_{2} k \sin \theta}\right]^{2} \\
\times & {\left[-k h_{1}+k x^{2} \cos ^{2} \theta_{m}\left(2 h_{1}-h_{2}\right)\right.} \\
& \left.+k \cos ^{4} \theta\left(-h_{1}+h_{2}-h_{3}\right)\right]^{2}
\end{aligned}
$$

where

$$
x=\frac{\cos \theta}{\cos \theta_{m}}
$$

and

$$
\theta_{m}=\frac{\pi}{2}\left(1 \pm \frac{1}{2 Q_{T}}\right)
$$

In 5(c), $Q_{T}$ is the total quality factor $(Q)$ of the filter and $\theta_{m}$ is the electrical length of the coupled stage at the passband edge. It is important to note that a variable $k$ is purposely introduced to (5a). It provides one extra degree of freedom in matching the coefficients in (5a) with those in (3) and preserves the designated Chebyshev response at the same time.

Substituting $N=2$ into (3), we have

$$
\frac{P_{o}}{P_{L}}=1+\varepsilon^{2}\left(2 x^{2}-1\right)^{2} .
$$

Matching (5a) with (6) and enforcing $k h_{1}=1, k \cos ^{2} \theta_{m}\left(2 h_{1}-\right.$ $\left.h_{2}\right)=2$ and $h_{2}=h_{1}+h_{3}$, we have

$$
\begin{aligned}
S_{1} & =2 \\
2 T_{2}^{2}-\frac{T_{1}^{4}}{2} & =\frac{1}{k} \\
\left(S_{1}+S_{2}\right)\left[S_{1} T_{1}^{2}-2\left(S_{1}+S_{2}\right)\right] & =-\frac{2}{k \cos ^{2} \theta_{m}} .
\end{aligned}
$$

Inserting (7) into (5a) yields

$$
\frac{P_{o}}{P_{L}}=1+\left[\frac{1}{2 T_{1}^{2} T_{2} k \sin \theta}\right]^{2}\left[\mathbf{T}_{2}(x)\right]^{2} .
$$

The term $\left[\mathbf{T}_{2}(x)\right]^{2}$ will provide an equal-ripple response in the passband, and its coefficient $\left[2 T_{1}^{2} T_{2} k \sin \theta\right]^{-1}$ not only defines the ripple level, but also implies a transmission zero at twice the design frequency, $2 f_{o}$, where $\theta=\pi$. Note that, in (7), there are five unknowns, i.e., $k, S_{1}, S_{2}, T_{1}$, and $T_{2}$, to be determined. By imposing the given ripple level to (8), the system has four conditions and there is one degree of freedom for choosing the solution. If in (5a) the variable $k$ is not introduced, however, the system will be fully determined.

\section{B. Filters of Order $N \leq 9$}

When $N$ is increased, the derivation is straightforward. The insertion loss function, however, is long and tedious when $N$ is large. One can utilize the structural symmetry to reduce the number of variables and times of matrix multiplication. The simultaneous conditions for solving $S_{i}$ and $T_{i}$ can be obtained by matching coefficients of the $\cos ^{n} \theta$ terms of the insertion 
loss function with those of $\left[\mathbf{T}_{N}(x)\right]^{2}$. Here, we derive the insertion-loss functions for filters of order $N \leq 9$, and the results are

$$
\begin{aligned}
\frac{P_{o}}{P_{L}}=1+\left[\frac{\cos \theta_{m} S_{1}\left(S_{1}^{2}-T_{1}^{2}\right)}{2 T_{1}^{2} \sin \theta}\right]^{2}\left[\mathbf{T}_{1}(x)\right]^{2}, & \\
N & =1 \\
\frac{P_{o}}{P_{L}}=1 & +\left[\frac{1}{2 T_{1} \cdot T_{2} \cdots T_{N+1} k \sin \theta}\right]^{2}\left[\mathbf{T}_{N}(x)\right]^{2}, \\
& \leq N \leq 9 .
\end{aligned}
$$

The simultaneous conditions are listed in Table I. It is found that $S_{1}=2$ holds for each $N$, and it is also valid for the maximally flat filters [8]. Note that total number of unknowns $\left(S_{i}\right.$ and $\left.T_{i}\right)$ for such an $N$ th-order filter is $2 \times[N / 2]+2$, where $[N / 2]$ is defined as the maximal integer no larger than $N / 2$. The number of conditions, including the specified ripple level, is less than that of unknowns when $N \geq 4$. In addition, the variable $k$ introduces an extra degree of freedom in matching the coefficients. It can be validated that the degrees of freedom for $N \leq 9$ are $[N / 2]$. This under-determined feature is very useful for circuit realization.

\section{RIPPLE LEVEL $R$ AND FRACTIONAL BANDWIDTH $\Delta$}

\section{A. Ripple Level Specified by $\theta_{m}$}

In the conventional synthesis [3], [4], the edges of the passband of a Chebyshev bandpass filter in (9) and (10) are defined by $|x|=1$. If this idea is used, the so-called ripple-level condition can be obtained by evaluating the functions in (9) and (10) at $\theta=\theta_{m}$ in (5c). It leads to

$$
\begin{aligned}
T_{1}^{2} & =\frac{\cot \theta_{m}\left(4-T_{1}^{2}\right)}{\sqrt{10^{\frac{R}{10}}-1}}, \quad N=1 \\
T_{1} \cdot T_{2} \cdots T_{N+1} & =\frac{1}{2 k \sin \theta_{m} \sqrt{10^{\frac{R}{10}}-1}}, \quad 2 \leq N \leq 9 .
\end{aligned}
$$

The in-band equal-ripple characteristic in (10) is expected to be provided by the Chebyshev function $\left[\mathbf{T}_{N}(x)\right]^{2}$ if its coefficient is a constant. The ripple levels within the passband, however, will be altered by the frequency-dependent term $1 / \sin \theta$. Note that for an $N$ th-order Chebyshev bandpass filter, there are $N$ transmission poles $\left(\left|S_{21}\right|^{2}=1\right)$ and $N-1\left|S_{11}\right|$ peaks $\left(\left|S_{21}\right|^{2}=\mathrm{min}\right)$ in the passband. Since the square of each $\mathbf{T}_{N}(x)$ is symmetric about $x=0$, unequal in-band $\left|S_{21}\right|$ ripples will occur when $N \geq 4$. If a small bandwidth decrement is acceptable, part of the altered ripple can be recovered. To tackle this, a method for correcting the ripple is formulated as follows.

\section{B. Ripple Level Specified by $\theta_{d}$}

The frequencies $\theta_{d}$ where peaks of ripples (in-band minima of $\left|S_{21}\right|$ or peaks of $\left.\left|S_{11}\right|\right)$ occur can be obtained by

$$
\frac{d}{d x}\left[\mathbf{T}_{N}(x)\right]=0 .
$$

Given the $R$ value, one can validate that the result is identical to that in (11) when $N=1$. When $N \geq 2$, the ripple condition derived from (13) can be written as

$$
T_{1} \cdot T_{2} \cdots T_{N+1}=\frac{1}{2 k \sin \theta_{d} \sqrt{10^{\frac{R}{10}}-1}}
$$

where

$$
\theta_{d}=\cos ^{-1}\left(\cos \frac{i \pi}{N} \cos \theta_{m}\right)
$$

for $i=1,2, \ldots, N-1$. The ripple $\left(\left|S_{11}\right|\right)$ peaks will increase when $\theta_{d}$ moves away from the passband edge $\theta=\theta_{m}$. For example, when $N=4$, there are three ripple peaks with two levels in the passband. Fig. 2 shows the detailed $\left|S_{21}\right|$ responses of three fourth-order filters with $\Delta=50 \%$ and $R=1,0.5$, and $0.1 \mathrm{~dB}$. When $R=1$ and $0.5 \mathrm{~dB}$, the peak ripple levels at $\theta_{d}=\pi / 2$ (center frequency) are 0.93 and $0.46 \mathrm{~dB}$, respectively. When $R=0.1 \mathrm{~dB}$, the deviation seems negligible.

Obviously, application of (14) will lead to a decrement of bandwidth. The decrement depends on which $\theta_{d}$ is chosen. It is plausible to redefine the $\left|S_{21}\right|$ value at $\theta_{d}$ with $i=1$ as the ripple level of the filter since it has the maximal $\left|S_{21}\right|$ ripple within the passband and it results in the smallest bandwidth decrement. For example, when $N=5$, there are four $\left|S_{21}\right|$ minima symmetrically locating on both sides of $f_{o}$ with two ripple levels. Based on the $\theta_{d}$ condition (14) with $R=0.1 \mathrm{~dB}$, when designated bandwidths are $40 \%$ and $50 \%$, the calculated bandwidths are $39.7 \%$ and $49.6 \%$, respectively. All these bandwidth decrements can be negligible. Our further studies show that the dependence of the decrements on the designated bandwidths and $R$ value (from 0.1 to $3 \mathrm{~dB}$ ) is more or less the same for filters of orders $N \leq 9$. Thus, the $\theta_{d}$ condition will be used for the experimental demonstrations herein.

\section{SOLUTIONS AND EXPERIMENTS}

Based on the simultaneous equations in Table I, together with the ripple-level condition (12) or (14), the variables $S_{i}$ and $T_{i}$ of all coupled stages can be solved, and then the linewidth and gap size can be determined by $Z_{o e i}=\left(S_{i}+T_{i}\right) \times Z_{o} / 2$ and $Z_{\text {ooi }}=\left(S_{i}-T_{i}\right) \times Z_{o} / 2$. The solution procedure can be referred to [8] and is briefly described as follows. For example, a fifthorder filter has two degrees of freedom for choosing the solution. One can take one of the six variables $\left(S_{i}, T_{i}\right)$ and $k$ as sweep variables to solve the equations. Since highly coupled structures have been planned to substitute the end stages, in the solutionsearching procedure, one can relax the dimensions of the middle stages and locate all the difficulties to the end stages as much as possible. After all solutions with tough geometric parameters are removed, one obtains a solution range for each variable. The modal characteristic impedance design graph can be useful for the solution selection.

It is worth mentioning that change of $k$ will not alter the Chebyshev passband response, but will lead the coupled stages to have different modal characteristic impedances. Consider a filter with $N=5, \Delta=40 \%$ and $R=0.1 \mathrm{~dB}$. We can take $k$ and $T_{1}$ as sweep variables to solve the simultaneous equations. Fig. 3 plots the root loci of $Z_{o e}$ and $Z_{o o}$ of the second and third 
TABLE I

Simultaneous Conditions For Synthesis OF CHEBysheV FILTERS OF ORders $N=1-9$

\begin{tabular}{|c|c|c|}
\hline$N$ & Conditions & \begin{tabular}{|c|} 
Degrees of \\
Freedom \\
\end{tabular} \\
\hline 1 & (1) $S_{1}=2$ & 0 \\
\hline 2 & $\begin{array}{l}\text { (1) } S_{1}=2 \\
\text { (2) } 2 T_{2}^{2}-T_{1}^{4} / 2=1 / k \\
\text { (3) } U_{1}\left(U_{1}-T_{1}^{2}\right)=1 /\left(k \cos ^{2} \theta_{m}\right)\end{array}$ & 1 \\
\hline 3 & $\begin{array}{l}\text { (1) } S_{1}=2 \\
\text { (2) } 4 T_{2}^{2} U_{1}-T_{1}^{2}\left(2 T_{2}^{2}+S_{2} T_{1}^{2}\right)=3 /\left(k \cos \theta_{m}\right) \\
\text { (3) } S_{2} U_{1}\left(U_{1}-T_{1}^{2}\right)=1 /\left(k \cos ^{3} \theta_{m}\right)\end{array}$ & 1 \\
\hline 4 & $\begin{array}{l}\text { (1) } S_{1}=2 \\
\text { (2) } 2 T_{2}{ }^{4}-T_{1}^{4} T_{3}^{3} / 2=1 / k \\
\text { (3) } 2 T_{3}^{2} U_{1}^{2}+2 U_{1}\left(2 T_{2}^{2} U_{2}-T_{1}^{2} T_{3}^{2}\right)-\left(2 T_{2}^{2}+T_{1}^{2} U_{2}\right)^{2} / 2+2 T_{2}^{4}=8 /\left(k \cos ^{2} \theta_{m}\right) \\
\text { (4) } U_{1} U_{2}^{2}\left(U_{1}-T_{1}^{2}\right)=4 /\left(k \cos ^{4} \theta_{m}\right)\end{array}$ & 2 \\
\hline 5 & $\begin{array}{l}\text { (1) } S_{1}=2 \\
\text { (2) } 2 T_{2}^{2} T_{3}^{2}\left(2 U_{1}-T_{1}^{2}\right)-T_{1}^{4} T_{3}^{2} U_{2}+4 S_{3} T_{2}^{4}=5 /\left(k \cos \theta_{m}\right) \\
\text { (3) } U_{2}\left\{4 U_{1}\left[2 S_{3} T_{2}^{2}-T_{3}^{2}\left(T_{1}^{2}-U_{1}\right)\right]-S_{3} T_{1}^{4} U_{2}-4 S_{3} T_{1}^{2} T_{2}^{2}\right\}=20 /\left(k \cos ^{3} \theta_{m}\right) \\
\text { (4) } S_{3} U_{1} U_{2}^{2}\left(U_{1}-T_{1}^{2}\right)=4 /\left(k \cos ^{5} \theta_{m}\right)\end{array}$ & 2 \\
\hline 6 & $\begin{array}{l}\text { (1) } S_{1}=2 \\
\text { (2) } 2 T_{2}^{4} T_{4}{ }^{2}-T_{1}^{4} T_{3}^{4} / 2=1 / k \\
\text { (3) } 4 T_{2}^{2} U_{1} V_{1}-T_{1}^{4} U_{2}\left(T_{3}^{2} U_{3}+T_{4}^{2} U_{2} / 2\right)-2 T_{1}^{2}\left(T_{2}^{2} V_{1}+T_{3}^{4} U_{1}\right)+2 V_{9}=18 /\left(k \cos ^{2} \theta_{m}\right) \\
\text { (4) } 2 T_{4}^{2} U_{1}^{2} U_{2}^{2}-2 T_{1}^{2} U_{2}\left(T_{4}^{2} U_{1} U_{2}+2 T_{3}^{2} U_{1} U_{3}+T_{2}^{2} U_{3}^{2}\right)+U_{2} U_{3}\left(4 T_{3}^{2} U_{1}^{2}-T_{1}^{4} U_{2} U_{3} / 2+4 T_{2}^{2} U_{1} U_{2} U_{3}\right)=48 /\left(k \cos ^{4} \theta_{m}\right) \\
\text { (5) } U_{1} U_{2}{ }^{2} U_{3}{ }^{2}\left(U_{1}-T_{1}^{2}\right)=16 /\left(k \cos ^{6} \theta_{m}\right)\end{array}$ & 3 \\
\hline 7 & $\begin{array}{l}\text { (1) } S_{1}=2 \\
\text { (2) } 4 T_{2}^{2} T_{4}^{2} V_{3}-T_{1}^{4} T_{3}^{2} V_{2}-2\left(T_{1} T_{2} T_{3} T_{4}\right)^{2}=7 /\left(k \cos \theta_{m}\right) \\
\text { (3) } 4 V_{2}\left(2 T_{2}^{2} U_{1} U_{3}-T_{1}^{2} V_{3}\right)-T_{1}^{4} U_{2} U_{3}\left(2 T_{3}^{2} S_{4}+T_{4}^{2} U_{2}\right)+4 T_{4}{ }^{2} T_{3}{ }^{2} U_{1}^{2} U_{2}+4 S_{4} V_{9}=56 /\left(k \cos ^{3} \theta_{m}\right) \\
\text { (4) } U_{2} U_{3}\left[8 S_{4} U_{1} V_{3}-4 T_{1}^{2}\left(T_{4}^{2} U_{1} U_{2}+T_{2}^{2} S_{4} U_{3}+2 T_{3}^{2} S_{4} U_{1}\right)-U_{2}\left(T_{1}{ }^{4} S_{4} U_{3}-4 T_{4}^{2} U_{1}^{2}\right)\right]=112 /\left(k \cos ^{5} \theta_{m}\right) \\
\text { (5) } S_{4} U_{1} U_{2}^{2} U_{3}^{2}\left(U_{1}-T_{1}^{2}\right)=16 /\left(k \cos ^{7} \theta_{m}\right)\end{array}$ & 3 \\
\hline 8 & 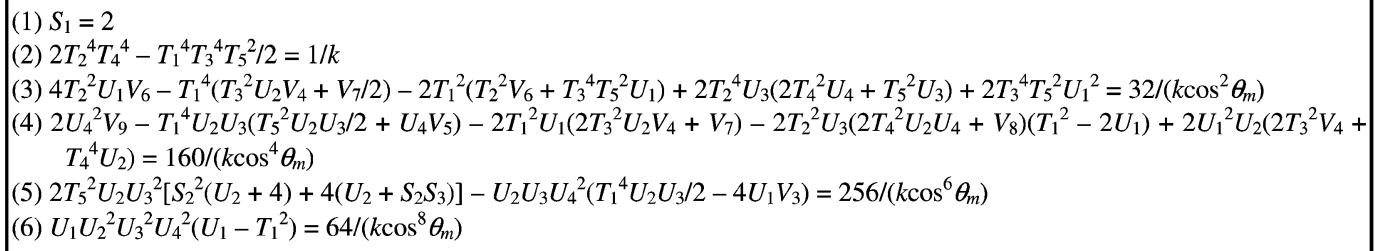 & 4 \\
\hline 9 & 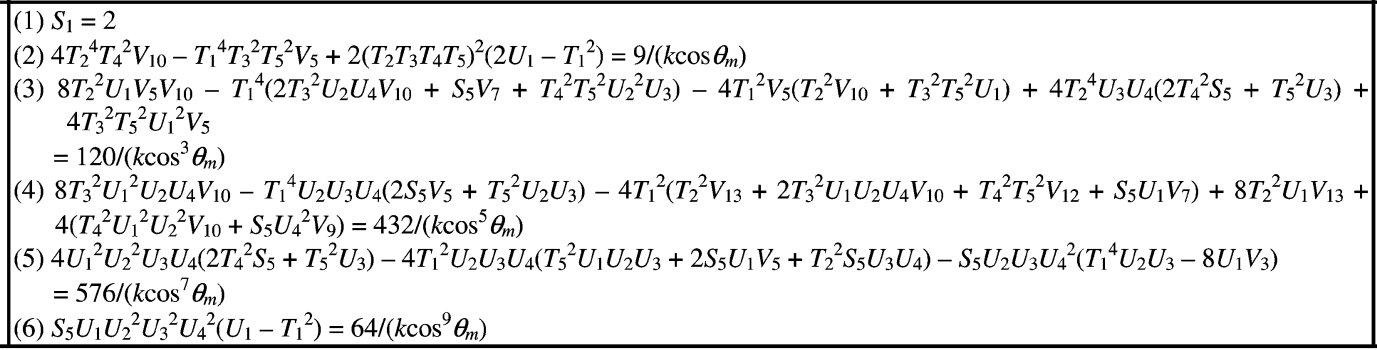 & 4 \\
\hline & $\begin{array}{l}U_{i}=S_{i}+S_{i+1}, i=1,2,3,4 \\
V_{1}=T_{3}^{2} U_{3}+T_{4}^{2} U_{2} \\
V_{2}=T_{3}^{2} S_{4}+T_{4}^{2} U_{2} \\
V_{3}=T_{2}^{2} U_{3}+T_{3}^{2} U_{1} \\
V_{4}=T_{4}^{2} U_{4}+T_{5}^{2} U_{3} \\
V_{5}=T_{3}^{2} U_{4}+T_{4}^{2} U_{2} \\
V_{6}=T_{3}^{2} V_{4}+T_{4}^{4} U_{2} \\
V_{7}=T_{3}^{4} U_{4}^{2}+T_{4}^{4} U_{2}^{2} \\
V_{8}=T_{3}^{2} U_{4}^{2}+T_{5}^{2} U_{2} U_{3} \\
V_{9}=T_{2}^{4} U_{3}^{2}+T_{3}^{4} U_{1}^{2} \\
V_{10}=T_{4}^{2} S_{5}+T_{5}^{2} U_{3} \\
V_{11}=S_{3}\left(S_{3}+2 S_{4}\right)\left(S_{5} U_{2}+S_{2} S_{4}\right)+S_{4}\left(S_{3} S_{4} S_{5}+S_{3} U_{3}^{2}+S_{2} S_{4} U_{4}\right) \\
V_{12}=S_{2}\left(S_{2}+2 S_{3}\right)\left(S_{4} U_{1}+2 S_{3}\right)+S_{3}\left(S_{2} S_{3} S_{4}+S_{2} U_{2}^{2}+2 S_{3} U_{3}\right) \\
V_{13}=2 T_{4}^{2} S_{5} U_{2} U_{3} U_{4}+T_{5}^{2} V_{11}+T_{3}^{2} S_{5} U_{3} U_{4}^{2}\end{array}$ & \\
\hline
\end{tabular}

stages for $k=0.5,1$, and 2 . The loci indicate that most of the solutions fall into the fan area of $G / d \geq 0.1$ and $W / d \geq 0.1$.
As $T_{1}$ is increased, the values of $Z_{o e 2}$ and $Z_{o o 2}$ increase, while those of $Z_{o e 3}$ and $Z_{o o 3}$ decrease. In this particular ex- 


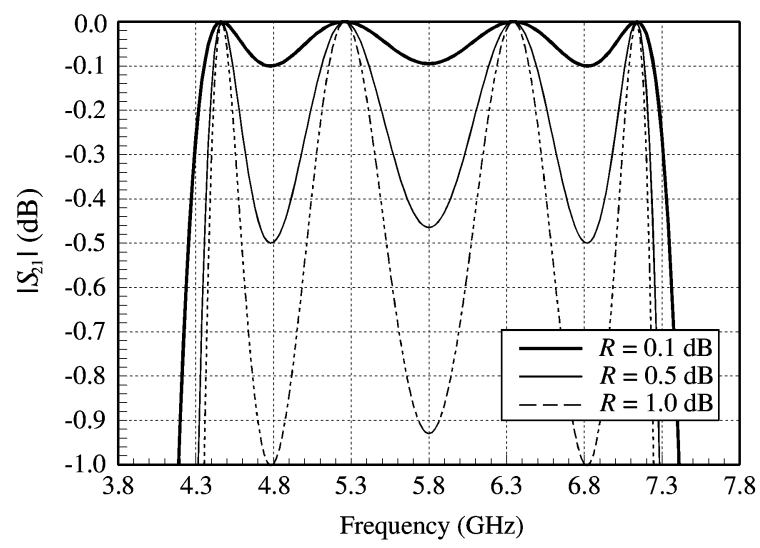

Fig. 2. Detailed $\left|S_{21}\right|$ responses in the passbands of fourth-order filters with $\Delta=50 \%$ and $R=0.1,0.5$, and $1 \mathrm{~dB}$.

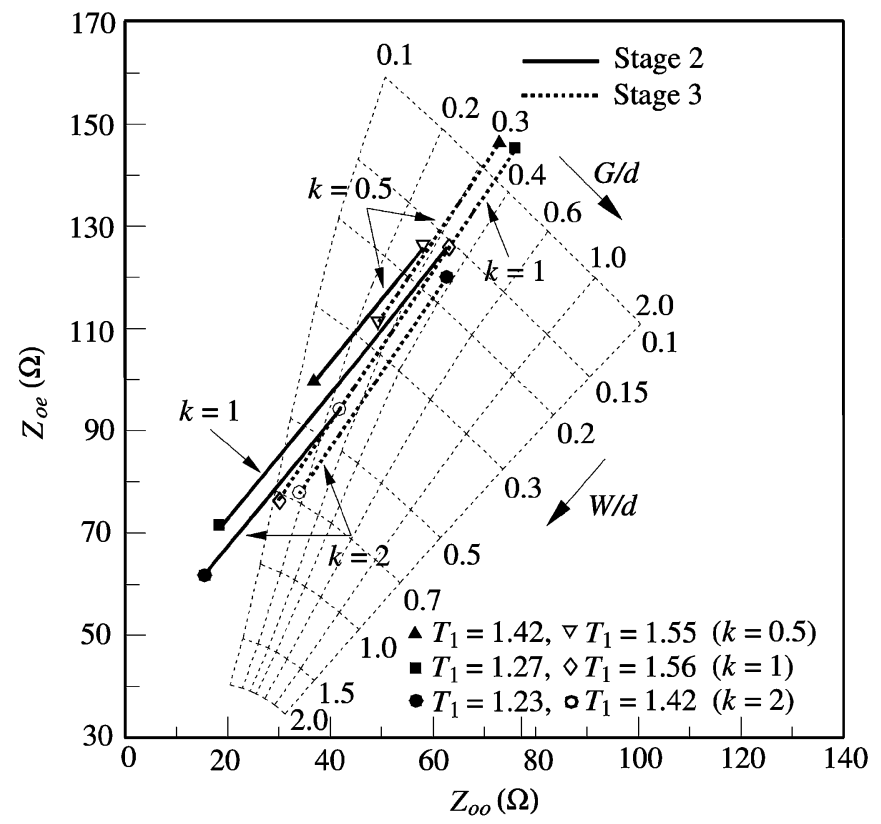

Fig. 3. Root loci of $Z_{o e}$ and $Z_{\text {oo }}$ of the second and third stages of fifth-order Chebyshev filters $(\Delta=40 \%$ and $R=0.1 \mathrm{~dB}$ ) with $k=0.5,1$, and 2 . Substrate: $\varepsilon_{r}=10.2$, thickness $d=1.27 \mathrm{~mm}$.

ample, the case $k=2$ has the easiest circuit dimensions for realization.

Table II investigates the changes of the modal impedances of the coupled stages from the conventional synthesis to the proposed method for fifth-order filters with a $0.1-\mathrm{dB}$ ripple and $\Delta$ ranging from $10 \%$ to $50 \%$. The listed solutions for the proposed method are selected since they are relatively easy to realize. In comparison of the listed data for $\Delta \leq 20 \%$, one can see that middle stages of the proposed method have much narrower dimensions than those of the conventional synthesis. In addition, the $Z_{o e} / Z_{o o}$ ratio of the end stages of the proposed method is much larger than that of the conventional synthesis. Note that, from Fig. 3, a larger $Z_{o e} / Z_{o o}$ ratio corresponds to a tighter gap size. The situation becomes even worse when the bandwidth is increased. For the conventional synthesis, the $Z_{o e} / Z_{o o}$ ratios are increased to around three only for the first stage when bandwidth is more than $40 \%$. Table II also compares the bandwidth
TABLE II

MODAl CHARACTERISTIC IMPEDANCES OF THE COUPLED STAGES OF FIFTH-ORDER CHEBYSHEV FILTERS BY THE PROPOSED METHOD AND CONVENTIONAL SYNTHESIS

\begin{tabular}{|c|c|c|c|c|c|c|c|}
\hline \multicolumn{2}{|c|}{$\begin{array}{c}N=5, \\
R=0.1 \mathrm{~dB}\end{array}$} & \multicolumn{3}{|c|}{ Proposed Method } & \multicolumn{3}{|c|}{ Coventional Synthesis } \\
\hline$\Delta$ & $i$ & $Z_{o e}(\Omega)$ & $Z_{o o}(\Omega)$ & $\begin{array}{l}\text { Simulated } \\
\text { BW(\%) }\end{array}$ & $Z_{o e}(\Omega)$ & $Z_{o o}(\Omega)$ & $\begin{array}{c}\text { Simulated } \\
\text { BW(\%) }\end{array}$ \\
\hline \multirow{3}{*}{$10 \%$} & 1 & 72.48 & 27.52 & \multirow{3}{*}{9.7} & 75.35 & 38.34 & \multirow{3}{*}{9.5} \\
\hline & 2 & 119.5 & 96.75 & & 57.05 & 44.52 & \\
\hline & 3 & 128.7 & 106.56 & & 55.23 & 45.68 & \\
\hline \multirow{3}{*}{$20 \%$} & 1 & 79.93 & 20.07 & \multirow{3}{*}{19.4} & 89.87 & 37.53 & \multirow{3}{*}{16.3} \\
\hline & 2 & 119.55 & 79.15 & & 65.67 & 40.61 & \\
\hline & 3 & 120.73 & 82.37 & & 61.37 & 42.28 & \\
\hline \multirow{3}{*}{$30 \%$} & 1 & 79.07 & 20.93 & \multirow{3}{*}{29.1} & 102.6 & 38.49 & \multirow{3}{*}{24.8} \\
\hline & 2 & 70.5 & 29.5 & & 75.85 & 38.27 & \\
\hline & 3 & 121.57 & 73.68 & & 68.42 & 39.78 & \\
\hline \multirow{3}{*}{$40 \%$} & 1 & 80.64 & 19.36 & \multirow{3}{*}{38.2} & 114.40 & 40.39 & \multirow{3}{*}{30.5} \\
\hline & 2 & 61.8 & 15.4 & & 87.61 & 37.5 & \\
\hline & 3 & 119.69 & 62.51 & & 76.38 & 38.2 & \\
\hline \multirow{3}{*}{$50 \%$} & 1 & 87.46 & 12.54 & \multirow{3}{*}{47.5} & 125.62 & 42.87 & \multirow{3}{*}{38} \\
\hline & 2 & 95 & 29.85 & & 100.93 & 38.3 & \\
\hline & 3 & 110.79 & 43.56 & & 85.25 & 37.53 & \\
\hline
\end{tabular}

decrements of the proposed method and the conventional synthesis. When $\Delta=20 \%$ is given, the conventional synthesis has $\Delta=16.3 \%$, while the proposed method has $\Delta=19.4 \%$. When $\Delta=50 \%$ is specified, the bandwidth of the conventional synthesis lacks of $12 \%$, while the bandwidth decrement of the proposed method is only $2.5 \%$.

Table III lists the even- and odd-mode characteristic impedances of coupled stages of seventh-order Chebyshev filters with $R=0.04$ and $0.1 \mathrm{~dB}$ and bandwidths from $30 \%$ to $50 \%$. Again, these data are selected because of they are relatively easy to realize by the standard PCB process.

Herein, the RT/Duroid 6010 high-frequency laminate $\left(\varepsilon_{r}=\right.$ 10.2 and thickness $d=1.27 \mathrm{~mm}$ ) is used as the circuit substrate. For demonstration, two filters $(\alpha$ and $\beta$ ) of order 5 with $\Delta=30 \%$ and $R=0.1 \mathrm{~dB}$ and one filter $(\chi)$ of order 9 with $\Delta=40 \%$ and $R=0.04 \mathrm{~dB}$, all designed at $f_{o}=2.45 \mathrm{GHz}$, are fabricated and measured. Ansoft's software package High Frequency Structure Simulator (HFSS) [14] is used for EM simulation.

1) Filters $\alpha$ and $\beta(N=5, \Delta=30 \%, R=0.1 \mathrm{~dB})$ : When $N=5$, six variables have to be solved for the three leading stages. Given $\Delta=30 \%$, we have $\theta_{d}=1.335 \mathrm{rad}$. The solution $S_{1}=2, T_{1}=1.163, S_{2}=2, T_{2}=0.82, S_{3}=3.905, T_{3}=$ 0.958 , and $k=4$ is chosen for demonstration. The $Z_{o e i}$ and $Z_{\text {ooi }}$ values of each stage are listed in Table IV. The modal characteristic impedances of the end stages are $Z_{o e 1}=79.07 \Omega$ and $Z_{o o 1}=20.93 \Omega$, and the corresponding linewidth and gap size for a usual coupled-line stage are 0.921 and $0.021 \mathrm{~mm}$, respectively. Note that the gap size is much smaller than the best resolution of the standard microstrip technology. If the bandwidth is larger or the ripple is smaller, the gap size will be even tougher for fabrication.

An aperture in the ground plane can be created under the coupled lines to enhance the coupling [9]. It is due to the aperture, more field lines originating from one of the coupled lines terminate to the other so that the coupling can be enhanced. By treating a section of such coupled lines as a four-port, numerical data such as the $Z$-parameters can be obtained by Ansoft's 
TABLE III

Modal Characteristic Impedances $(\Omega)$ OF Coupled Stages of SEventh-Order Chebyshev Filters

\begin{tabular}{|c|c|c|c|c|c|c|c|c|c|c|}
\hline \multirow{2}{*}{ Order } & \multirow{2}{*}{$\begin{array}{c}\Delta \\
(\%)\end{array}$} & \multirow{2}{*}{$\begin{array}{c}R \\
(\mathrm{~dB})\end{array}$} & \multicolumn{2}{|c|}{ 1st stage } & \multicolumn{2}{|c|}{ 2nd stage } & \multicolumn{2}{|c|}{ 3rd stage } & \multicolumn{2}{|c|}{ 4th stage } \\
\hline & & & $Z_{o e}$ & $Z_{\text {oo }}$ & $Z_{o e}$ & $Z_{\circ o}$ & $Z_{o e}$ & $Z_{o o}$ & $Z_{o e}$ & $Z_{\text {oo }}$ \\
\hline \multirow{6}{*}{$N=7$} & \multirow{2}{*}{30} & 0.04 & 81.05 & 18.95 & 73.33 & 30.43 & 121.15 & 72.65 & 135.79 & 80.01 \\
\hline & & 0.1 & 85.03 & 14.97 & 123.8 & 73.7 & 79.25 & 38.95 & 104.87 & 64.43 \\
\hline & \multirow{2}{*}{40} & 0.04 & 87.83 & 12.18 & 102.73 & 40.13 & 123.25 & 58.7 & 132.03 & 64.78 \\
\hline & & 0.1 & 84.44 & 15.56 & 91.9 & 36.55 & 117.83 & 56.83 & 143.38 & 73.18 \\
\hline & \multirow{2}{*}{50} & 0.04 & 86.19 & 13.81 & 87.65 & 34.65 & 91.9 & 39.7 & 121.79 & 62.51 \\
\hline & & 0.1 & 86.54 & 13.46 & 85.58 & 31.18 & 70 & 23.5 & 79.48 & 34.82 \\
\hline
\end{tabular}

TABLE IV

Solutions and Modal Characteristic Impedances of the Stages of the Three EXPERIMENTAL Filters

\begin{tabular}{|l|l|l|l|l|l|}
\hline \hline \multicolumn{1}{|c|}{ Filters } & \multicolumn{1}{c|}{ 1st stage } & \multicolumn{1}{c|}{ 2nd stage } & \multicolumn{1}{c|}{ 3rd stage } & 4th stage & \multicolumn{1}{c|}{ 5th stage } \\
\hline \hline$\alpha$ and $\beta$ & $S_{1}=2$ & $S_{2}=2$ & $S_{3}=3.905$ & & \\
$N=5$ & $T_{1}=1.163$ & $T_{2}=0.82$ & $T_{3}=0.958$ & Same as the 3rd & Same as the 2nd \\
$\Delta=30 \%$ & $Z_{o e 1}=79.07 \Omega$ & $Z_{o e 2}=70.5 \Omega$ & $Z_{o e 3}=121.57 \Omega$ & stage & stage \\
$R=0.1 \mathrm{~dB}$ & $Z_{o o 1}=20.93 \Omega$ & $Z_{o o 2}=29.5 \Omega$ & $Z_{o o 3}=73.68 \Omega$ & & \\
\hline \hline$\chi$ & $S_{1}=2$ & $S_{2}=2.636$ & $S_{3}=2.984$ & $S_{4}=2.955$ & $S_{5}=4.014$ \\
$N=9$ & $T_{1}=1.47$ & $T_{2}=1.123$ & $T_{3}=1.043$ & $T_{4}=1.091$ & $T_{5}=1.241$ \\
$\Delta=40 \%$ & $Z_{o e 1}=86.75 \Omega$ & $Z_{o e 2}=93.98 \Omega$ & $Z_{o e 3}=100.68 \Omega$ & $Z_{o o 4}=101.15 \Omega$ & $Z_{o e 5}=131.38 \Omega$ \\
$R=0.04 \mathrm{~dB}$ & $Z_{o o 1}=13.25 \Omega$ & $Z_{o o 2}=37.83 \Omega$ & $Z_{o o 3}=48.53 \Omega$ & $Z_{o o 4}=46.6 \Omega$ & $Z_{o o 5}=69.33 \Omega$ \\
\hline
\end{tabular}

HFSS. The matrix pencil method [15] can then be used to accurately extract its phase constants and characteristic impedances. The electrical length of each stage is a quarter-wave long at frequency $f_{o}$, which is the geometric mean of the even- and odd-mode phase velocities. To eliminate the open-end fringing effect, the EM simulator HFSS is employed to finely tune the length of each coupled stage. In (1), the modal phase velocities of all coupled lines are assumed identical. Its purpose is to simplify the derivation of the conditions listed in Table I. In practice, it is well known that different modal phase velocities will cause spurious $\left|S_{21}\right|$ response at $2 f_{o}$. We have proposed several approaches, e.g., [16] and [17], to tackle this problem. In the proposed synthesis, it is worth mentioning the assumption that equal modal phase velocities have a negligible effect on the accuracy of passband response and bandwidth.

Fig. 4 compares the measured $\left|S_{11}\right|$ and $\left|S_{21}\right|$ responses with the calculated results. The dashed curves are the theoretical data obtained by (10). In the experimental circuit, the aperture has a length identical to that of the coupled lines and a width of $7.45 \mathrm{~mm}$. Its width is chosen wider than the distance between the outer edges of the coupled line to assure a sufficiently high coupling level. The measured data show good agreement with the simulation. Compared the theoretical prediction, the measurement is short of only $0.9 \%$ bandwidth. The detailed measured data show that the in-band $\left|S_{21}\right|$ is only $1.1 \mathrm{~dB}$, which is acceptable for many applications. The ripple level of the $\left|S_{11}\right|$ curve is better than $14 \mathrm{~dB}$, in contrast to the theoretical value $16.4 \mathrm{~dB}$. The group delay in the passband is from 2 to $4 \mathrm{~ns}$.

The end stages of the above fifth-order filter are also realized by three-line microstrip sections [10]. The three metal strips are equally spaced $\left(G_{1}\right)$ and have identical linewidths $\left(W_{1}\right)$. By constructing a design graph like Fig. $3\left(\varepsilon_{r}=10.2\right)$, the geometric parameters of the sections can be determined. Fig. 5(a)

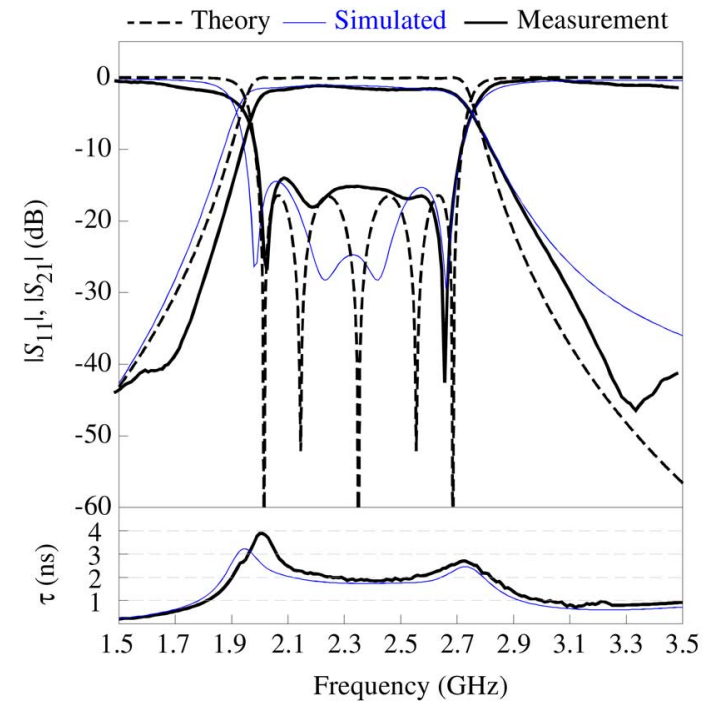

Fig. 4. Measured, simulated, and theoretical responses of filter $\alpha$. End coupled stages are realized by a coupled-line section with a ground-plane aperture. Circuit dimensions: $W_{1}=2.75 \mathrm{~mm}, G_{1}=0.15 \mathrm{~mm}, W_{2}=0.97 \mathrm{~mm}$, $G_{2}=0.16 \mathrm{~mm}, W_{3}=0.15 \mathrm{~mm}, G_{3}=0.724 \mathrm{~mm}$. Substrate: $\varepsilon_{r}=$ 10.2 , thickness $=1.27 \mathrm{~mm}$.

plots the measured data together with the calculated responses. The practical $\left|S_{11}\right|$ and $\left|S_{21}\right|$ responses show even better agreement with the calculation than the previous demonstration. The in-band $\left|S_{21}\right|$ is also only $0.6 \mathrm{~dB}$, and the peak $\left|S_{11}\right|$ is $16.6 \mathrm{~dB}$. The measurement is, nevertheless, still short of $0.8 \%$ bandwidth. Fig. 5(b) shows a photograph of the experimental circuit.

The reason why the three-line circuit has better in-band $\left|S_{21}\right|$ performance and improved $\left|S_{11}\right|$ ripples could be due to the fact that it has no aperture on the ground plane, which may cause 


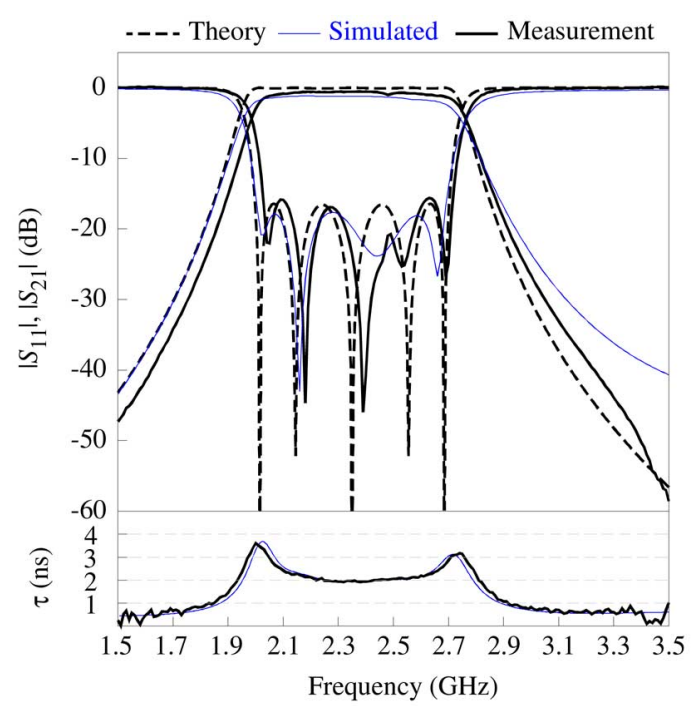

(a)

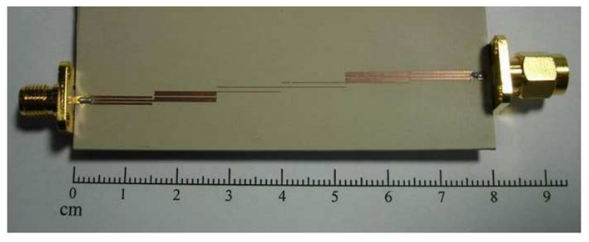

(b)

Fig. 5. (a) Measured, simulated, and theoretical responses of filter $\beta$. End coupled stages are realized by a three-line section. (b) Experimental circuit. $W_{2}, G_{2}, W_{3}$, and $G_{3}$ are identical to those in Fig. 4. Dimensions of the three-line stage: $W_{1}=0.475 \mathrm{~mm}, G_{1}=0.13 \mathrm{~mm}$.

power losses resulted from possible radiation. Note that the two substitutes for the end stages are required to implement identical coupling levels. Since the gap size of the three-line structure $(0.13 \mathrm{~mm})$ is smaller than that of the coupled lines with ground-plane aperture $(0.15 \mathrm{~mm})$, it is reasonable to deduce that the latter can offer higher coupling than the three-line structure when they have the same gap size. Of course, this deduction requires that the aperture width be large enough.

2) Filter $\chi(N=9, \Delta=40 \%, R=0.04 d B)$ : The third experiment uses the microstrip-to-CPW broadside-coupled sections [11] to implement the end stages. Fig. 6 plots the structure of the broadside-coupled lines. To make the design simpler, the width of the microstrip is made identical to that of the active conductor of the CPW to reduce the number of geometric parameters. The modal parameters of this asymmetric coupled line are extracted in a way similar to the two previous cases. Some transmission line characteristics of the broadside-coupled lines can be referred to [13].

For a ninth-order filter, there are ten variables of five coupled sections to be solved. Given $\Delta=40 \%$ and $R=0.04 \mathrm{~dB}$, the solution $S_{1}=2, T_{1}=1.47, S_{2}=2.636, T_{2}=1.123, S_{3}=$ $2.984, T_{3}=1.043, S_{4}=2.955, T_{4}=1.091, S_{5}=4.014$, and $T_{5}=1.241$ for $k=1$ is chosen for the experiment. This solution is chosen owing to that realization of all the middle sections is possible (minimal linewidth $=0.13 \mathrm{~mm}$ ). Note that the end stages have to imitate a microstrip coupled stage with $Z_{o e 1}=86.75 \Omega$ and $Z_{o o 1}=13.25 \Omega$; the linewidth $W_{1}=$ $0.72 \mathrm{~mm}$ and gap size $G_{1}=0.001 \mathrm{~mm}$ if a usual coupled-

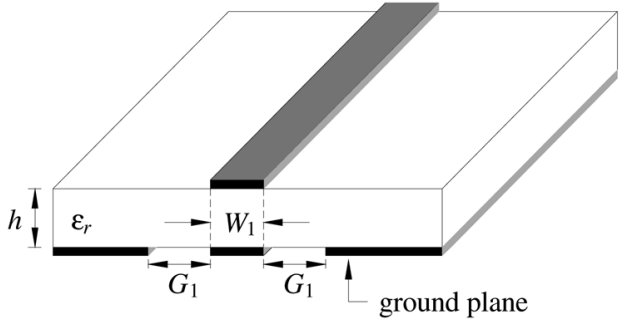

Fig. 6. Microstrip-to-CPW broadside-coupled lines.

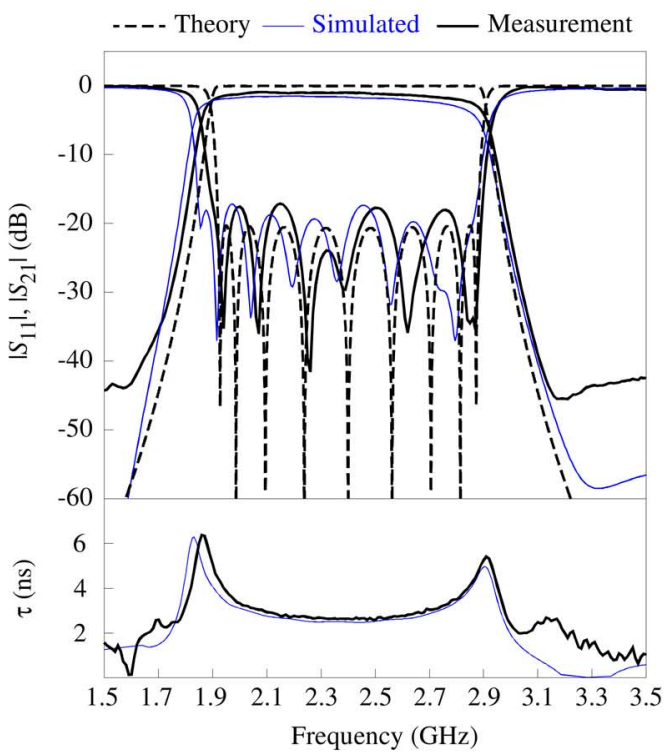

(a)

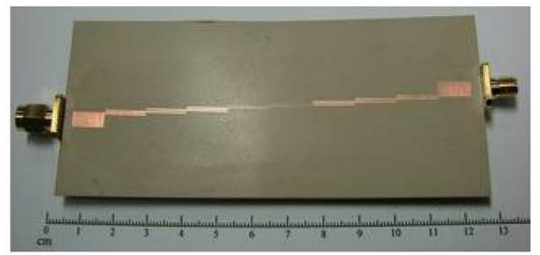

(b)

Fig. 7. (a) Theoretical, simulated, and measured responses of the ninth-order filter $\chi$. (b) Fabricated circuit (top view). Dimensions: $W_{1}=4.4 \mathrm{~mm}, G_{1}=$ $1.38 \mathrm{~mm}, W_{2}=0.52 \mathrm{~mm}, G_{2}=0.22 \mathrm{~mm}, W_{3}=0.37 \mathrm{~mm}, G_{3}=0.40 \mathrm{~mm}$, $W_{4}=0.38 \mathrm{~mm}, G_{4}=0.35 \mathrm{~mm}, W_{5}=0.13 \mathrm{~mm}, G_{5}=0.47 \mathrm{~mm}$.

line section is used. Fig. 7(a) plots the theoretical, simulated, and measured results. All of them show good agreement. The measured insertion loss is $0.99 \mathrm{~dB}$, and the measured bandwidth is only $0.2 \%$ more than the theoretical response by (10). The maximal measured in-band return loss is $17.1 \mathrm{~dB}$ in contrast to the theoretical value of $20.3 \mathrm{~dB}$. Fig. 7(b) is a photograph showing the top side of the experiment circuit.

Almost all microwave filters must be enclosed in a metal box in any practical application. For a given set of specifications, only the geometric parameters of the coupled stages need changing since the $Z_{o e i}$ and $Z_{o o i}$ (or $S_{i}$ and $T_{i}$ ) values specify the passband. The apertures on the ground plane may cause radiation, which may affect the performances of the filter. The measured results of filters $\alpha$ and $\chi$ indicate that the in-band $\left|S_{21}\right|$ are acceptable in most of practical applications. In the stopband, radiation could deteriorate the rejection level due to unwanted 
coupling from portions of the filter directly to the output terminal. One possible way to solve the problem is to block the unwanted coupling path.

\section{CONCLUSION}

New and rigorous synthesis formulas have been derived for parallel-coupled line filters with Chebyshev responses for orders up to nine. The technique accurately predicts the passband response and circuit bandwidth. Simultaneous equations and the ripple-level conditions have been formulated and tabulated for determining the circuit dimensions. When filter order $N \geq 4$, the passband function has unequal ripple levels resulted from the $1 / \sin \theta$ term in the derived insertion-loss expressions. Three structures suitable for realizing high coupling levels have been incorporated into the end stages of the three experimental circuits for validation of the formulation. All measured data show good performance and have good agreement with the theory and simulation.

\section{ACKNOWLEDGMENT}

The authors are grateful to the National Center for High Performance Computing, Hsinchu, Taiwan, R.O.C., for the HFSS software package and related services to this research.

\section{REFERENCES}

[1] S. B. Cohn, "Parallel-coupled transmission-line resonator filters," IRE Trans. Microw. Theory Tech., vol. MTT-6, no. 4, pp. 223-231, Apr. 1958.

[2] C.-Y. Chang and T. Itoh, "A modified parallel-coupled filter structure that improves the upper stopband rejection and response symmetry," IEEE Trans. Microw. Theory Tech., vol. 39, no. 2, pp. 310-314, Feb. 1991.

[3] G. L. Matthaei, L. Young, and E. M. T. Johns, Microwave Filters, Impedance Matching Networks, and Coupling Structures. Norwood, MA: Artech House, 1980

[4] D. M. Pozar, Microwave Engineering, 2nd ed. New York: Wiley, 1998.

[5] K.-S. Chin, L.-Y. Lin, and J.-T. Kuo, "New formulas for synthesizing microstrip bandpass filters with relatively wide bandwidths," IEEE Microw. Wireless Compon. Lett., vol. 14, no. 5, pp. 231-233, May 2004.

[6] J. M. Drozd and W. T. Joines, "Maximally flat quarter-wavelength-coupled transmission-line filters using $Q$ distribution," IEEE Trans. Microw. Theory Tech., vol. 45, no. 12, pp. 2100-2113, Dec. 1997.

[7] W. W. Mumford, "Tables of stub admittances for maximally flat filters using shorted quarter-wave stubs," IEEE Trans. Microw. Theory Tech., vol. MTT-13, no. 9, pp. 695-696, Sep. 1965.

[8] K.-S. Chin and J.-T. Kuo, "Insertion loss function synthesis of maximally flat parallel-coupled line bandpass filters," IEEE Trans. Microw. Theory Tech., vol. 53, no. 10, pp. 3161-3168, Oct. 2005.

[9] M. D. C. Velázquez-Ahumada, J. Martel, and F. Medina, "Parallel coupled microstrip filters with ground-plane aperture for spurious band suppression and enhanced coupling," IEEE Trans. Microw. Theory Tech., vol. 52, no. 3, pp. 1082-1086, Mar. 2004.

[10] J.-T. Kuo and E. Shih, "Wideband bandpass filter design with three line microstrip structures," Proc. Inst. Elect. Eng., vol. 149, no. 5, pt. H, pp. 243-247, Oct. 2002

[11] H. Wang, L. Zhu, and W. Menzel, "Ultra-wideband bandpass filter with hybrid microstrip/CPW structure," IEEE Microw. Wireless Compon. Lett., vol. 15, no. 12, pp. 844-846, Dec. 2005.

[12] J.-T. Kuo, Y.-C. Chiou, and J.-S. Wu, "Miniaturized rat race coupler with microstrip-to-CPW broadside-coupled structure and steppedimpedance sections," in IEEE MTT-S Int. Microw. Symp. Dig., Honolulu, HI, Jun. 2007, pp. 169-172.
[13] Y.-C. Chiou, J.-T. Kuo, and J.-S. Wu, "Miniaturized dual-mode ring resonator bandpass filter with microstrip-to-CPW broadside-coupled structure," IEEE Microw. Wireless Compon. Lett., vol. 18, no. 2, pp. 97-99, Feb. 2008.

[14] High Frequency Structure Simulator (HFSS). ver. 9.2, Ansoft, Pittsburgh, PA, 2004

[15] Z. A. Mariievic, T. K. Sarkar, Y. Hua, and A. R. Djordjevic, "Timedomain measurements with the Hewlett-Packard network analyzer HP 8510 using the matrix pencil method," IEEE Trans. Microw. Theory Tech., vol. 39, no. 3, pp. 538-547, Mar. 1991.

[16] J.-T. Kuo and M.-H. Wu, "Corrugated parallel-coupled line bandpass filters with multispurious suppression," IET Microw. Antennas Propag., vol. 1, no. 3, pp. 718-722, Jun. 2007.

[17] J.-T. Kuo, U.-H. Lok, and M.-H. Wu, "Novel corrugated coupled-line stage with ideal frequency response and its application to bandpass filter design with multi-harmonic suppression," in IEEE MTT-S Int. Microw. Symp. Dig., Honolulu, HI, Jun. 2007, pp. 553-556.

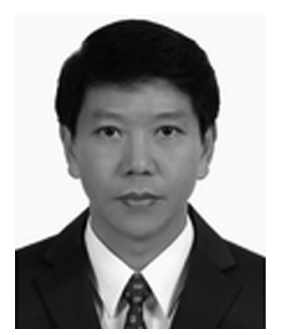

Kuo-Sheng Chin (S'05-M'06) received the M.S.E.E. degree from Syracuse University, Syracuse, NY, in 1993, and the Ph.D. degree in communication engineering from National Chiao-Tung University, Hsinchu, Taiwan, R.O.C., in 2005.

In 1986, he joined the Chung Shan Institute of Science and Technology, Tao-Yuan, Taiwan, R.O.C., as Research Assistant, becoming an Assistant Scientist and then an Associate Scientist. He is currently an Assistant Professor with the Department of Electronic Engineering, Chang Gung University, Tao-Yuan, Taiwan, R.O.C. His main research interests are microwave measurements, microwave circuits, radome and antenna designs, and electromagnetic pulse (EMP) research

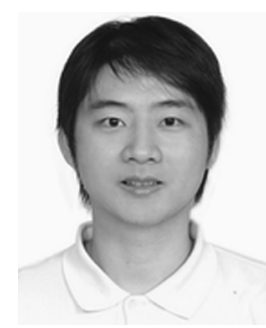

Yi-Chyun Chiou ( $\mathrm{S}^{\prime}$ 04) was born in Taoyuan, Taiwan, R.O.C., on September 25, 1979. He received the B.S. and M.S. degrees in electronic engineering from Feng Chia University, Taichung, Taiwan, R.O.C., in 2001 and 2003, respectively, and is currently working toward the Ph.D. degree in communication engineering at National Chiao Tung University (NCTU), Hsinchu, Taiwan, R.O.C.

From 2003 to 2004, he was a Lecturer with the Department of Electronic Engineering, Nan Kai Institute of Technology, Nanto, Taiwan, R.O.C. His research interests include the design of microwave devices and RF modules for microwave and millimeter-wave applications.

Mr. Chiou was a corecipient of the 2007 Asia-Pacific Microwave Conference (APMC) Prize.

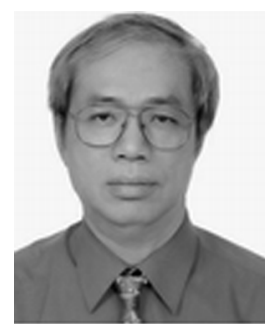

Jen-Tsai Kuo (S'88-M'92-SM'04) received the $\mathrm{Ph} . \mathrm{D}$. degree from the Institute of Electronics, National Chiao Tung University (NCTU), Hsinchu, Taiwan, R.O.C., in 1992.

Since 1984, he has been with the Department of Communication Engineering, NCTU, where he is currently a Professor. From 1995 to 1996, he was a Visiting Scholar with the Electrical Engineering Department, University of California at Los Angeles (UCLA). Since February 2007, he has been the Secretary General of NCTU. His research interests include analysis and design of microwave integrated circuits and numerical techniques in electromagnetics.

Dr. Kuo is currently an Editorial Board member of the IEEE TRANSACTIONS ON Microwave THEORY AND TECHNiQues and the IEEE Microwave AND WIRELESS COMPONENTS LETTERS. He was a corecipient of the 2002 Best Paper Award presented at the National Telecommunication Conference, Taiwan, R.O.C., and a corecipient of the 2007 Asia-Pacific Microwave Conference (APMC) Prize. He was the recipient of the Taiwan Citation Laureate 2006 presented by Thomson Scientific and the 2007 Distinguished Research Award presented by the National Science Council, Taiwan, R.O.C. 